\title{
Epigallocatechin-3-gallate Improves Chronic Alcohol Induced Cognitive Dysfunction in Rats by Interfering with the Neuro-inflammatory, Cell Death and Oxido-nitrosative Stress Pathways
}

\section{Ankit Uniyal}

IIT BHU: Indian Institute of Technology BHU Varanasi

Akhilesh Kotiyal

IIT BHU: Indian Institute of Technology BHU Varanasi

Anagha Gadepalli

Indian Institute of Technology Banaras Hindu University: Indian Institute of Technology BHU Varanasi Obulapathi Ummadisetty

Indian Institute of Technology Banaras Hindu University: Indian Institute of Technology BHU Varanasi Vinod Tiwari ( $\nabla$ vtiwari.phe@iitbhu.ac.in )

IIT BHU: Indian Institute of Technology BHU Varanasi https://orcid.org/0000-0002-8491-2255

\section{Research Article}

Keywords: Alcohol, Cell Death, Dementia, Epigallocatechin-3-gallate, Nuclear Factor kappa $\beta$, Oxidativenitrosative stress.

Posted Date: April 19th, 2021

DOl: https://doi.org/10.21203/rs.3.rs-248882/v1

License: (c) (i) This work is licensed under a Creative Commons Attribution 4.0 International License. Read Full License

Version of Record: A version of this preprint was published at Metabolic Brain Disease on August 12th, 2021. See the published version at https://doi.org/10.1007/s11011-021-00794-5. 


\section{Abstract}

Alcohol consumption for a longer period of time is linked with neuronal damage and an increase in inflammatory signaling resulting in cell death and dementia. Natural compounds are the focus of research due to their high efficacy and good safety profile. Here we have investigated the effect of chronic epigallocatechin-3-gallate (EGCG) administration against the alcohol-induced cognitive deficit rats. Male Wistar rats were exposed to the ethanol $(10 \mathrm{~g} / \mathrm{kg}$; oral gavage) for ten weeks and treated with EGCG (25, 50 , and $100 \mathrm{mg} / \mathrm{kg}$ ) for the same duration. Ethanol exposure led to the impaired spatial memory and learning in rats assessed using the Morris water maze and elevated plus-maze test. Further, we assessed the role of EGCG in mitigating the oxidative stress, neuroinflammatory and cell death signaling associated markers. Co-administration with EGCG significantly prevented all the behavioral, biochemical and molecular alterations in the different brain regions of ethanol-treated rats in a dose-dependent manner. EGCG suppressed the acetylcholinesterase activity, increased oxidative-nitrosative stress, cytokines (TNF-alpha and IL-1 beta), NF-kappa $\beta$ and caspase-3 levels in both the cortex and hippocampus of ethanol-treated rats. Our preliminary study demonstrated that EGCG improves the oxidonitrosative stress, inflammation, and cell death signaling associated with ethanol-induced cognitive dysfunction. This suggests the potential role of EGCG in mitigating the cognitive deficits associated with chronic alcohol consumption.

\section{Introduction}

Alcohol use disorder (AUD) is one of the leading causes of death worldwide. An estimated survey has reported that 95 million people are dependent on alcohol (Castillo-Carniglia et al. 2019). The prevalence of AUD is $34.2 \%$ among US population (Fan et al. 2019). Heavy alcohol consumption cause damage to the peripheral and central nervous system. The peripheral pathophysiology contributes to the development of somatosensory disorders. Whereas, damage to the central nervous system leads to a variety of brain disorders among which cognitive decline is prominent (Mende 2019; Slayday et al. 2020). Magnetic resonance imaging (MRI) with diffusive tensor imaging studies has suggested a reduced volume and microstructural damage to the white matter regions (Crespi et al. 2019, 2020). There are no well-established mechanisms of alcohol-induced brain toxicity although various hypotheses and pathways have been proposed by researchers. The most common pathways include neuronal excitotoxicity due to affected neurotransmission, free radical production leading to increased oxidative stress and edema due to imbalanced cellular ion transport control (Sanchez-Alavez et al. 2019; Xu et al. 2019). Thus, regardless of the well-proven existence of alcoholic dementia and neuronal damage, the mechanisms behind are still poorly understood.

The brain is highly dependent on glucose for ATP generation which allows it to perform various physiological functions. In the normal physiological process, the mitochondria generate ATP by the phosphorylation process which involves generation of reactive oxygen species (ROS). The increased ROS level cause oxidative stress to occur and to compensate this the initiation of enzymatic activity of the anti-oxidant system takes place. If the anti-oxidant system fails to suppress the risen stress due to some 
pathological conditions, then damage to the brain can occur. Alcohol induces heavy ROS generation that leads to the neuronal damage, the reasons behind this consideration are; firstly the high consumption of oxygen by brain; secondly free radical damage prone polyunsaturated fatty acids are found in higher concentration in brain; and finally the relative oxidant defense system of the brain is very poor (Bhatia et al. 2019). Apart from these explanations the presence of iron and ascorbate molecules also contributes to the more ROS generation by enabling the Fenton/Haber Weiss reaction within the specific brain regions (Gandhi and Abramov 2012). The exceeded oxidative stress in the brain cause other defense mechanisms to get activated and as a result, the process of cell death starts affecting the DNA integrity, the function of proteins, and virtue of membrane lipids, cumulatively these events cause brain damage (Islam 2017; Simpson and Oliver 2020). Ethanol gets oxidized into the acetaldehyde in the liver by cytochrome P-450 which generates the free radicals and increases lipid peroxidation, also it depletes the endogenous antioxidants (glutathione, catalase, superoxide dismutase, vitamin E and ascorbate) (Ambade and Mandrekar 2012).

The oxidative stress induced inflammation is also another major contributor to the pathophysiology of alcohol induced brain damage (Sanchez-Alavez et al. 2019). The effectiveness of antioxidants in cognitive enhancement and their protective effect on neuronal damage associated with memory impairment is well explored (Ahmad et al. 2019). Epigallocatechin-3-gallate (EGCG) is the major catechin found in green tea, it carries a strong antioxidant effect with additional potent anti-inflammatory and neuromodulatory effects (Ahadi et al. 2019; Xu et al. 2020). Various preclinical studies have suggested that the EGCG prevents the development of dementia like condition that is linked with Alzheimer disease (Wei et al. 2016; Xicota et al. 2017). Moreover, EGCG was found to be neuroprotective and to slow down the neurodegeneration by inhibiting the neuronal cell death pathways (He et al. 2015; Xu et al. 2017). Present study was designed to explore the mechanisms behind the alcohol-induced brain damage and associated cognitive deficit, and to investigate the therapeutic effect of EGCG.

\section{Methodology}

\subsection{Animals}

Adult male Wistars rats (200-250 gm) were used in this study. Animals were kept under standard housing conditions; $12 \mathrm{hr}$ light/dark cycle, $25 \pm 5^{\circ} \mathrm{C}$ temperature and full access to the food and water. The acclimatization was performed by placing rat cages to the laboratory environment before conducting experiments. All the tests were performed between 9.00 to $17.00 \mathrm{hrs}$ and were executed in accordance with the guidelines of Committee for Control and Supervision of Experimentation on Animals (CPCSEA), Government of India on animal experimentation. We have selected the sample size as per our previously published literature and as well as same work directions from other laboratories as indicated below (Tiwari \& Chopra, 2011, 2013). The Institutional Animal Ethics Committee (IAEC) of Banaras Hindu University has approved all the procedures and protocols (IAEC/2566).

\subsection{Experimental design}


We had randomized all the 36 animals into six experimental groups. Each group consisted of six rats $(\mathrm{n}=$ $6 /$ group). The group I rats was administered distilled water per oral; rats in group II were given per oral $10 \mathrm{~g} / \mathrm{kg}$ of ethanol; rats in group III, IV, V were administered with ethanol and the test drug EGCG $(25,50$ and $100 \mathrm{mg} / \mathrm{kg}$ respectively per oral). The EGCG dissolved in distilled water and administered orally in rats at different dosage $(25,50$ and $100 \mathrm{mg} / \mathrm{kg})$. For per se comparison the Group VI was added in which rats received EGCG (100 mg/kg; per oral). The EGCG was administered daily $1 \mathrm{hr}$ before ethanol administration for ten weeks. On the week 6,8 and 10 of study, the behavioral task was performed and the data were analyzed by a blind observer. At the end of 10th week of treatment period the rats were sacrificed using high dose anesthesia. The rat brains were collected in a separate suite at the same time of the day during their active cycle. Rats were decapitated following an intraperitoneal injection of ketamine $300 \mathrm{mg} / \mathrm{kg}$ with $30 \mathrm{mg} / \mathrm{kg}$ xylazine. Furthermore, the brains were rapidly removed and placed on dry ice for isolation of cerebral cortex and hippocampus. of the brain was done to isolate the hippocampus and cortex. Samples were stored at $-80^{\circ} \mathrm{C}$ for further analysis.

\subsection{Behavioral tests}

\subsubsection{Morris water maze test}

The method for Morris water maze task was obtained from previous described procedure (Morris et al. 1982). The task was performed with the minor modification for testing the spatial memory of rats on week 6, 8 and 10. The maze was circular tank like arena of a diameter of $180 \mathrm{~cm}$ and height of $60 \mathrm{~cm}$, filled with water up to $40 \mathrm{~cm}\left(28.5 \pm 2^{\circ} \mathrm{C}\right.$ ). A platform (diameter $12.5 \mathrm{~cm}$ ) was placed into the arena in such a way that it merges slightly under water so that the platform became invisible to the rats. Bright colored cues were marked in the room so that they are easily seen from the pool. The cue presentation was done to get used by rats to navigate the spatial orientation. The training trial lasted for five days and to each day they were given four consecutive training trails. During the training trial, the rats were placed into the tank on water surface heading to the wall. For the next subsequent training trial, the rats were again placed in a similar manner starting from different quadrants of the maze the sequence of quadrants was randomly selected. Once rats found the hidden platform, they are allowed to remain on to the platform for $20 \mathrm{~s}$. Every trial lasted for $90 \mathrm{~s}$ each supplemented with an inter-period of $30 \mathrm{~s}$. After the completion of the training trial, the test trials occur in which was performed in a similar manner to the training trial. The escape latency (s) was determined by measuring the time taken to reach the platform.

\subsubsection{Memory consolidation test}

The modification was performed in Morris water maze to assess the consolidation of memory as per previously described method (Kuhad et al. 2009). The platform was removed from the arena and probe trial was made run. The consolidation of memory was assessed on 10th week and time spent by rats in target quadrant was taken as a measure of it.

\subsubsection{Elevated plus maze (EPM) test}


On the 6th, 8th and 10th week of study the evaluation of memory acquisition and retention was performed in EPM. Although the result of EPM may be confounded by anxiety in animals. EPM comprised of two open and two closed arms making a plus sign shape. For acquisition memory initial transfer latency (ITL) was determined by placing the rats at the center of plus sign heading its face toward the open arm and the time taken to enter the close arm was measured. For retention memory, the test was repeated after $24 \mathrm{~h}$ in a similar manner and time to enter the closed arm was calculated as a measure of retention time latency (RTL). The RTL values were expressed as the percentage of ITL (Sharma and Gupta 2002).

\subsubsection{Closed field activity}

On the 10th week, the digital photoactometer was used to assess the closed field activity. The test has been performed to exclude out the possible interference of locomotor behavior on the parameters of learning and memory (Fernandes and Gupta 2019). The values were expressed as the number of count $/ 5 \mathrm{~min}$.

\subsection{Tissue harvesting and processing}

The hippocampus and cortex were isolated from rat brain tissues and stored for further analysis. The samples were incubated for $30 \mathrm{~min}$ in a solution mixture of ice-cold hypotonic buffer $(1 \mathrm{ml})$, detergent (1\%) and $1 \mathrm{mM}$ Dithiothreitol. Centrifugation was performed for the processed samples at 10,000 rpm for $10 \mathrm{~min}$ at a cold temperature of $4^{\circ} \mathrm{C}$. The cytosolic fraction was separated out by decantation and pellets were re-suspended using the nuclear lysis buffer (100 $\mu$ l) and vortexed. The centrifugation at 14,000 rpm for $10 \mathrm{~min}$ at a cold temperature of $4^{\circ} \mathrm{C}$ was performed for processed samples. The nuclear fraction was separated out by decantation. Both the cytosolic and nuclear fractions were stored at $-80^{\circ} \mathrm{C}$ for further analysis of the oxido-nitrosative assays and as well as for the estimation of IL- $1 \beta, T N F-a, N F K \beta$, and Caspase-3.

\subsection{Biochemical estimations}

\subsubsection{Quantification of hippocampal and cortical acetylcholinesterase activity}

The hippocampal and cortical acetylcholinesterase quantification was done by the method discussed previously (Ellman et al. 1961). The calculations were performed with the molar extinction coefficient of the chromophore $(1.36 \times 104 \mathrm{M}-1 \mathrm{~cm}-1)$. Values were presented as the percentage of control.

\subsubsection{Quantification of hippocampal and cortical lipid peroxidation (LPO)}

Assay for LPO estimation was performed using the earlier described method (Uniyal et al. 2019).The LPO was determined by quantifying the malondialdehyde (MDA) level. In brief, $0.5 \mathrm{ml}$ of hippocampal and cortical cytosolic sample were incubated with Tris- $\mathrm{HCl}(0.5 \mathrm{ml})$ for $2 \mathrm{~h}\left(37^{\circ} \mathrm{C}\right)$. Further the addition of $1 \mathrm{ml}$ 
of $10 \%$ trichloroacetic acid was performed. After that the 10 min centrifugation was done at $1000 \mathrm{~g}$. The separation of supernatant was done and to this $1 \mathrm{ml}$ of thiobarbituric acid was mixed and boiled for 10 min. The samples were cooled and distill water $(1 \mathrm{ml})$ was added to them. Finally, we performed a spectrophotometric analysis at $532 \mathrm{~nm}$. Calculations were performed and final values were presented as $\mathrm{nmol}$ of MDA/mg protein. Levels of protein were determined by Biuret method.

\subsubsection{Quantification of hippocampal and cortical reduced glutathione activity (GSH)}

Assay for determining the GSH enzymatic activity was performed as per the procedure described by Jollow et al. (Jollow et al. 1974). In brief, $1.0 \mathrm{ml}$ of sulphosalicylic acid (4\%) was used to precipitate the hippocampal and cortical cytosolic fraction. The sample incubation was performed for $1 \mathrm{~h}$ at a temperature of $4^{\circ} \mathrm{C}$. Further, the $15 \mathrm{~min}$ centrifugation was done at $1200 \mathrm{~g}$ and liquid above the solid pellet residue was decanted. The decanted liquid was then mixed with $0.1 \mathrm{mM} 0.2 \mathrm{ml}$ Ellman's reagent, $\mathrm{pH}$ 8.0 and $0.1 \mathrm{M} 2.7 \mathrm{ml}$ phosphate buffer $\mathrm{pH}$ 7.4. As soon as the yellowish color appears the spectrophotometric analysis was performed at $512 \mathrm{~nm}$. Values were presented as GSH levels $\mu \mathrm{moles} / \mathrm{mg}$ protein.

\subsubsection{Quantification of hippocampal and cortical of superoxide dismutase activity (SOD)}

The assay for SOD was performed in accordance with the previously described method by Kono et al. (Kono 1978). In brief, the mixture containing $50 \mathrm{mM}$ sodium carbonate, $0.1 \mathrm{mM}$ EDTA, and $96 \mathrm{mM}$ of nitro blue tetrazolium was taken in the cuvette. Further, $0.01 \mathrm{ml}$ cytosolic fraction was added to the above mixture along with $0.05 \mathrm{ml}$ of hydroxylamine hydrochloride ( $\mathrm{pH} \mathrm{6.0)}$. Finally, the reaction was observed for 2 min by spectrophotometric analysis at $560 \mathrm{~nm}$ with an interval of $30 \mathrm{~s}$.

\subsubsection{Quantification of hippocampal and cortical of catalase activity}

The catalase assay was done with reference to the earlier developed method given elsewhere (Claiborne 2018). Briefly, the final $3.0 \mathrm{ml}$ assay mixture containing the $0.05 \mathrm{ml}$ cytosolic fraction sample, $1.0 \mathrm{ml}$ $\mathrm{H}_{2} \mathrm{O}_{2}(0.019 \mathrm{M})$ and $1.95 \mathrm{ml}$ phosphate buffer $(0.05 \mathrm{M}, \mathrm{pH} 7.0)$ was prepared. The spectrophotometric analysis was performed to determine the change in optical density at $240 \mathrm{~nm}$. The calculations were performed and results were presented in ${\mathrm{k} \mathrm{min}^{-1}}^{1}$.

\subsubsection{Quantification of hippocampal and cortical of Nitrite}

Nitrite assay was done as per the previously described method (Sharma et al. 2018). Greiss reagent was utilized as an indicator of nitric oxide synthesis. The calculation was performed and results were presented as nitrite levels $\mu \mathrm{g} / \mathrm{mg}$ protein. 


\subsubsection{Quantification of hippocampal and cortical of IL-1 $\beta$ and TNF-alpha}

The assay was performed on hippocampal and cortical homogenates as per manufacturer instructions using R\&D Systems Quantikine Rat IL-1 $\beta$ and TNF-a ELISA kits.

\subsubsection{Quantification of hippocampal and cortical of NF kappa $\beta$ p65 unit}

That assay was performed on hippocampal and cortical homogenate as per manufacturer instruction using NF-K $\beta /$ p65 Active ELISA (Imgenex, San Diego, USA) ELISA kit.

\subsubsection{Quantification of hippocampal and cortical Caspase-3 using colorimetric assay}

A colorimetric assay was performed for the estimation of caspase-3 levels. The protease activity was measured in tissue homogenates samples using a caspase specific peptide conjugated with a chromophore ( $p$-nitroaniline). The peptide cleavage done by caspase was analyzed by using the spectrophotometric technique at $405 \mathrm{~nm}$. The percentage of control was taken as a measure to express the results.

\subsection{Statistical analysis}

The data was interpreted by one-way ANOVA or two-way ANOVA followed by multiple comparisons with Tukey's post hoc analysis or Bonferroni's correction. The statistical analysis was performed by GraphPad Prism Software version 8. Results were presented as mean \pm SD and $P<0.05$ value was considered as statistically significant.

\section{Results}

\subsection{Behavioral assessment interpretations}

\subsubsection{Chronic EGCG treatment improved ethanol-associated cognitive dysfunction in the Morris water maze test}

The escape latency was measured during training trial on the 6th, 8 th and 10 th week. The ethanol administration in rats produced a significant $F(30,168)=60.15$; $D F=30, M S, 229.4, p<0.0001)$ increase in time to find the platform as compared to control group rats indicating the cognitive deficit. Whereas, the EGCG $(25,50$ and $100 \mathrm{mg} / \mathrm{kg})$ treated rats were observed to have significant $(\mathrm{p}<0.05)$ reduction in escape latency time. (Fig. 1.A). In probe trial a significant difference was observed across all the groups. The time spent in the target quadrant was found to be significantly $(p<0.001)$ reduced in the rats administered with ethanol as compared to the control group rats. The EGCG $(25,50$ and $100 \mathrm{mg} / \mathrm{kg})$ 
treatment significantly $(p<0.05)$ escalated the time spent in the target quadrant in rats that are administered with chronic ethanol. However, EGCG per se was observed to have a negligible effect in the probe trial (Fig. 1.B). These results indicate the protective effect of EGCG on alcohol induced cognitive decline.

\subsubsection{Chronic EGCG treatment improved learning and memory of rats with chronic ethanol administration in Elevated plus maze test}

We had observed no significant variation in initial transfer latency across all the groups. However, the ethanol group showed a significant $(p<0.05)$ increase in the retention transfer latency $(R T L)$ when compared to the control group. Moreover, a significant $[(F(10,72)=52.48 ; D F=10, M S, 931.5, p<0.0001)$ reduction in RTL was found with the EGCG $(25,50$ and $100 \mathrm{mg} / \mathrm{kg})$ treatment in ethanol administered rats indicating its memory-enhancing the effect (Fig. 1C).

\subsubsection{Chronic EGCG treatment had no effect on locomotor activity of rats}

At the end of the 10th week, no significant difference was observed across all the groups for locomotor activity. The control, ethanol administered, EGCG (25, 50 and 100) treated and per se group, were having the mean values $223.33 \pm 8.1,231.2 \pm 11.2,239.86 \pm 12.7,252.4 \pm 15.5$ and $211.57 \pm 5.4,214.67 \pm 5.84$ respectively. These results demonstrate that the locomotor activity of rats was not affected with treatment.

\subsection{Biochemical observations and interpretation}

\subsubsection{Chronic EGCG treatment decreased hippocampal and cortical acetylcholinesterase activity in rats with chronic ethanol administration}

A Significantly increased acetylcholinesterase activity was observed in hippocampal (2.28 fold) and cortical (3.66 fold) brain regions of rats with chronic ethanol administration as compared with respective samples from control group. A significant effect across the groups was observed on acetylcholinesterase activity in hippocampus and cerebral cortex after one-way ANOVA followed by Brown-Forsythe test [(F $(5,24)=27.57 ; D F=5, p<0.0001),(F(5,24)=80.39 ; p<0.0001)$ respectively. The EGCG $(25,50$ and 100 $\mathrm{mg} / \mathrm{kg}$ ) treatment significantly $(\mathrm{p}<0.001)$ decreased acetylcholinesterase activity dose-dependently in both brain areas of rats with chronic ethanol exposure. Whereas, EGCG per se group had a negligible effect on acetylcholinesterase activity of the brain as compared with control group. (Fig. 2A, 2B). 


\subsubsection{Chronic EGCG treatment enhances hippocampal and cortical antioxidant enzymatic activity in rats with chronic ethanol administration}

Ethanol chronic exposure in rats significantly $(p<0.05)$ reduces the hippocampal and cortical enzymatic activity of catalase as compared to the rats in the control group A significant effect across the groups was observed on catalase activity in hippocampus and cerebral cortex after one-way ANOVA followed by Brown-Forsythe test $[(F(5,24)=53.47 ; D F=5, p<0.0001),(F(5,24)=96.98 ; p<0.0001)$ respectively (Fig. 2C, 2D). A similar trend was observed in levels of other major anti-oxidant enzymes GSH was shown in cerebral cortex and hippocampus was shown in the (Fig. 2E, 2F) A significant effect across the groups was observed on GSH activity in hippocampus and cerebral cortex after one-way ANOVA followed by Bartlett's test $[(F(5,24)=21 ; D F=5, p<0.0001),(F(8,24)=115.8 ; D F=5 ; p<0.0001)$ respectively, however in the case of SOD in the cerebral cortex after one-way ANOVA followed by Bartlett's test $[(F(5,24)=51$; $D F=5, p<0.0001),(F(5,24)=167.5 ; D F=5 ; p<0.0001)$ respectively, was shown (Fig. 2G, 2H). Moreover, chronic treatment with EGCG $(25,50$ and $100 \mathrm{mg} / \mathrm{kg})$ significantly reverses these enzymatic activities in hippocampus and cortex brain areas of rats with ethanol administration $(p<0.0001)$.

\subsubsection{Chronic EGCG treatment suppressed hippocampal and cortical nitrosative stress and LPO levels in rats with chronic ethanol administration}

The ethanol administration for chronic-duration significantly increases the nitrite levels in hippocampus ( 1.78 fold) and cortex ( 1.89 fold) brain region of rats. A significant effect across the groups was observed on nitrile activity in hippocampus and cerebral cortex after one-way ANOVA followed by Bartlett's test [( $F$ $(5,24)=113.28 ; D F=5, p<0.0001),(F(5,24)=88.07 ; D F=5 ; p<0.0001)$ respectively, Moreover, EGCG (25, 50 and $100 \mathrm{mg} / \mathrm{kg})$ treatments significantly $(p<0.0001)$ decreased the nitrite levels in the brain of chronic ethanol administered rats. This effect was dose-dependent. However, EGCG per se group rats were observed to have a negligible effect on brain nitrite levels. (Fig. 3A, 3B). Rats administered with chronic alcohol were found to have significant $(p<0.001$ increase in lipid peroxidation in the hippocampus (3.57 fold) and cerebral cortex (3.51 fold) as compared to the control group rats. A significant effect across the groups was observed on LPO activity in hippocampus and cerebral cortex after one-way ANOVA followed by Bartlett's test $[(F(5,24)=75.44 ; D F=5, p<0.0001),(F(8,24)=112.8 ; D F=5 ; p<0.0001)$ respectively. The EGCG treated $(25,50$ and $100 \mathrm{mg} / \mathrm{kg})$ rats were observed to have a significant $(p<0.001)$ reduction in lipid peroxidation in both brain regions of ethanol administered rats. Moreover, the EGCG per se group rats were observed to have a negligible effect on lipid peroxidation in the brain. (Fig. 3C, 3D).

\subsubsection{Chronic EGCG treatment mitigates hippocampal and cortical tumor necrosis factor-alpha TNF-a in rats with}




\section{chronic ethanol administration}

Chronic ethanol administration in rats significantly $(p<0.05)$ increased the hippocampal $(3.47 \mathrm{fold})$ and cortical (3.17 fold) TNF-a levels as compared to the control group rats. A significant effect across the groups was observed on TNF- $a$ expression in hippocampus and cerebral cortex after one-way ANOVA followed by Bartlett's test $[(F(5,24)=320.8 ; D F=5, p<0.0001),(F(8,24)=418.8 ; D F=5 ; p<0.0001)$ respectively, EGCG $(25,50$ and $100 \mathrm{mg} / \mathrm{kg})$ treatment decreases the TNF-a levels significantly $(p<0.05)$ in both the brain regions of ethanol administered rats dose dependently. Whereas, EGCG per se group rats were observed to have no change in the level of TNF-a in the rat brain. (Fig. 4A, 4B)

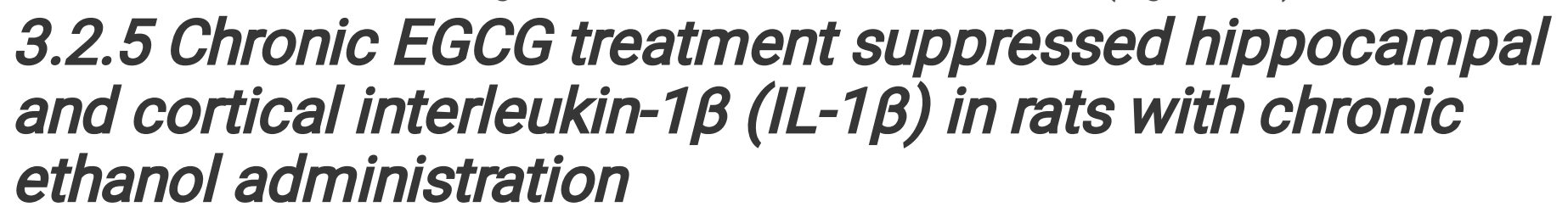

The IL-1 $\beta$ levels were significantly $(p<0.05)$ increased in the hippocampus $(4.33$ fold $)$ and cerebral cortex ( $4.79 \mathrm{fold}$ ) of rats with chronic ethanol administration when compared to the rats in control. A significant effect across the groups was observed on $I L-1 \beta$ expression in hippocampus and cerebral cortex after oneway ANOVA followed by Bartlett's test $[(F(5,24)=1270 ; D F=5, p<0.0001),(F(8,24)=326.8 ; D F=5 ; p<$ $0.0001)$ respectively. Moreover, rats treated with EGCG $(25,50$ and $100 \mathrm{mg} / \mathrm{kg})$ showed significant $(\mathrm{p}<$ 0.05 ) reduction in hippocampal and cortical IL-1 $\beta$ levels. This effect was dose-dependent. (Fig. 4C, 4D).

\subsubsection{Chronic EGCG treatment ameliorated hippocampal and cortical nuclear factor kappa beta (NF-Kß) p56 subunit levels in rats with chronic ethanol administration}

Rats administered with chronic ethanol were observed to have significantly $(p<0.05)$ elevated NF-K $\beta$ p65 subunit levels in the hippocampus ( 7.62 fold) and cerebral cortex ( 2.38 fold) when compared to the control group. The significant effect across the groups was observed on $N F-K \beta$ expression in hippocampus and cerebral cortex after one-way ANOVA followed by Bartlett's test $[(F(5,24)=42 ; D F=5, p$ $<0.0001),(F(8,24)=98 ; D F=5 ; p<0.0001)$ respectively. Treatment with EGCG $(25,50$ and $100 \mathrm{mg} / \mathrm{kg})$ significantly $(p<0.001)$ reduced the hippocampal and cortical NF-k $\beta$ p56 subunit expression of chronic ethanol administered rats and this effect was dose-dependent. Whereas, no effect was observed in EGCG per se treatment group on the expression of NFK $\beta$ p56. (Fig. 4E, 4F)

\subsubsection{Chronic EGCG treatment suppressed hippocampal and cortical caspase-3 levels of rats with chronic ethanol administration}


The rats administered with chronic ethanol were found to have a significant $(p<0.05)$ increase in caspase3 level in the hippocampus ( 3.62 fold) and cortex ( 4.15 fold) brain regions as compared to the rats in control group. The significant effect across the groups was observed on caspase- 3 expression in hippocampus and cerebral cortex after one-way ANOVA followed by Bartlett's test $[(F(5,24)=14 ; D F=5, p$ $<0.0001),(F(5,24)=3342 ; D F=5 ; p<0.0001)$ respectively. A significant $(p<0.05)$ reduction was observed with EGCG treatment $(25,50$ and $100 \mathrm{mg} / \mathrm{kg})$ in caspase-3 levels of hippocampus and cortex in a dosedependent mode. However, EGCG per se treatment was not found to have any effect on levels of Caspase-3 in rat brain. (Fig 4.G, 4H).

\section{Discussion}

Chronic alcohol consumption leads to brain cell damage and dementia. The clinicians fail to recognize the alcoholism as a causal factor in the majority of the patient and thus alcohol-related dementia remains under-diagnosed. The clinical features of alcohol-induced dementia closely resemble dementia associated with other causal subtypes and also it is not easy to confirm this type of dementia solely based on the patient's history. The emerging evidence suggests the contribution of ROS, nitrosative pathway, IL-1 $\beta$, and TNF- $a$ to modulate the NF-K $\beta$ signaling cascade and initiation of cell death signaling via caspase-3 modulation in hippocampal and cortical brain circuits of rats chronically exposed to ethanol. Results obtained in the current study support the hypothesis that ethanol administration for the chronic period in rat's leads to the oxidative stress-mediated inflammatory cascade which further initiates the cell death cascades and thus may involve in cognitive deficit. Moreover, we have observed that EGCG treatment suppressed the development of cognitive deficits by chronic ethanol exposure. The mechanism behind the therapeutic effect of EGCG is not only restricted to the suppression of oxido-nitrosative pathway but also extended to the attenuation of pro-inflammatory cytokines, NF-k $\beta$ and cell death mediator Caspase-3 in hippocampus and cortex brain areas of rats. Major classical manifestations associated with alcohol-induced dementia in the clinical scenario are memory impairment, language problem, and inefficiency to conduct complex motor tasks. Recently various reports have suggested the involvement of oxido-inflammatory cross-linked pathway and cell death cascade in the alcoholic brain associated cognitive deficit (Montesinos et al. 2015; Hernández et al. 2016; Sun et al. 2017). The shortterm memory impairments and spatial orientation impairments associated with alcoholic brain are thought to be correlated with the cholinergic neurotransmission. This aberration in the cognitive system was found to be improved with the administration of galantamine which is an acetylcholinesterase inhibitor thus acting by uplifting the levels of acetylcholine and ultimately strengthening the cholinergic neurotransmission (lliev et al., 1999; Liu et al., 2018). A recent report has suggested that the heavy alcohol consumption in rats leads to the reduced nuclei volume in the MPFC leading to the transient spatial and working memory decline (West et al. 2018).

The present study suggests that alcohol exposure for duration induces cognitive impairments in rats. A number of cumulative shreds of evidence indicates that the dietary intake of antioxidants can reverse age-related dementia. The EGCG treatment for a chronic duration significantly reversed the ethanolinduced memory impairments in rats. The probe trial interpretation also showed the disruption of memory 
in chronic alcohol administered rats. Moreover, this effect was significantly reversed by EGCG treatment. The alcohol induced oxidative stress is considered as key contributor to the pathophysiology of cognitive dysfunction. Moreover, it is well evident that oxidative stress is an imbalance of ROS generation and decreased activity of antioxidant enzymes. Targeting both the component could provide a potential therapeutic approach for the management of various CNS pathologies associated with cognitive decline. Various studies have supported the hypothesis that ethanol administration for a chronic duration raises the cellular oxidative stress which further disrupts the lipids, proteins, and DNA and ultimately causing the defects in cellular integrity (Tobore 2019; Petrella et al. 2020). Our results demonstrated that alcohol exposure leads to the increase in LPO and nitrite levels significantly, indicating the elevated oxidative stress. Moreover, the anti-oxidant enzymatic activity of GSH, SOD, and catalase was significantly suppressed in hippocampus and cortex brain areas of rats exposed to the chronic ethanol. Researchers have linked the activation of ROS producing enzymes by chronic ethanol administration leading to the increased ROS levels (Aboulmaouahib et al. 2018). Further, the present study demonstrated a significant reduction in the oxidative stress with EGCG treatment in hippocampus and cortex area of chronic ethanol treated rats brain suggesting the potent antioxidant effect of EGCG. Result of our study is in line with the observations from a previous study that shows the EGCG treatment attenuates the amyloid beta-induced neuronal damage and suppressed oxidative cascade linked cell death (Zhang et al. 2017). The nitrosative pathway is also considered to be involved in cognitive decline (Tiwari and Chopra 2013; Butterfield and Boyd-Kimball 2020). The peroxynitrite is a very strong oxidant that forms by the reaction between the superoxides and nitrite. This compound reacts with DNA, a variety of proteins and unsaturated fatty acids thereby performing the post-translation modifications and impairing energy homeostasis this compound leads to the neuronal cell damage (Ramdial et al. 2017). In present study, we have shown that the EGCG treatment for a chronic duration significantly suppressed the hippocampal and cortical nitrite levels of ethanol-induced cognitive deficit rats.

Acetylcholine by acting through the muscarinic receptors can induce glial cell proliferation and prevent cell death thus it acts as a trophic factor in the brain. A recent study has suggested that restoring the function of acetylcholine can improve the cognitive performance (Park et al. 2020). The present study indicates that chronic alcohol administration increases the levels of acetylcholinesterase in the rat brain thus minimizing the neuroprotective effect of acetylcholine. Whereas, the EGCG treatment reduced the acetylcholinesterase levels in the hippocampus and cortex region of the brain of alcohol administered rats.

Pro-inflammatory cytokine levels are increased with memory associated neuropathological conditions. Activation of neuroinflammatory signaling in rats with chronic ethanol administration, contribute to neuronal damage (Girard et al. 2019). Recently a report suggested that the chronic ethanol treated rats showed the persistent alterations of cytokines levels and altered microglia morphology providing the evidence of ethanol-induced neuroinflammation (Sanchez-Alavez et al. 2019). In our previous work we had demonstrated that the ethanol-associated cognitive decline is linked with increased II-1 $\beta$ and TNF-a levels in hippocampal and cortical brain regions of rats (Tiwari and Chopra 2011). The current study is in line with our previous findings, as we had observed the significantly increased IL-1 $\beta$ and TNF-a levels in 
both brain regions of chronic ethanol administered rats. Thus, it indicates that the hippocampal and cortical neuroinflammation is critically involved in the impairments of learning and memory. Moreover, this elevation in levels of IL-1 $\beta$ and TNF- $\alpha$ in both brain areas of ethanol-exposed rats was significantly reduced with the chronic EGCG treatment dose dependently. Activation of NF-kB unit stimulates the enzymes that are responsible for ROS production such as NADPH oxidase, NOS and COX2. These ROS producing enzymes are also up-regulated in chronic ethanol exposure thus there is a possible involvement of NF-kB activation leading to these oxidative events (Fukai and Ushio-Fukai 2020; Sun et al. 2020). Our result demonstrated a significantly higher level of hippocampal and cortical NFK $\beta$ as well as the caspase- 3 in ethanol-treated rats supporting the hypothesis that cell death pathway participates in the development of alcohol-associated cognitive decline (Dwivedi et al. 2018). Moreover, our results from the present study showed that the treatment with EGCG significantly suppressed the hippocampal and cortical levels of NFK $\beta$ and caspase- 3 in rats administered with chronic ethanol. Hence, our preliminary study provides directions that EGCG act through interfering with neuroinflammation, cell death and oxidative stress cascades. Further in-depth molecular studies are required to confirm the effect of EGCG on neuroinflammation, cell death and oxidative stress.

\section{Conclusion}

In a nutshell, our study demonstrated the EGCG treatment prevents the development of chronic ethanolassociated cognitive deficits by suppressing the oxido-nitrosative stress-dependent facilitation of cell death signaling in the brain. Hence, EGCG could be used as a potential therapeutic option for patients with alcoholic dementia or encephalopathy.

\section{Declarations}

\section{Disclosure/Conflict of Interest}

Authors have none to declare.

\section{Fundings}

This work is supported by Senior Research Fellowship awarded to Mr. Ankit Uniyal under the supervision of Dr. Vinod Tiwari by Indian Council of Medical Research (5/3/8/44/ITR-F/2019-ITR). This work is also supported by the Core Research Grant (CRG/2020/002621) and SPARC grant (SPARC/20182019/P435/SL) awarded to Dr. Vinod Tiwari by Science and Engineering Research Board and Ministry of Human Resource \& Development, Government of India respectively.

\section{Authors contribution}

The conceptualization of the study was done by V.T. Further, the idea was discussed with all authors. The experimental framework was designed by V.T., A.U. and Akhilesh. V.T., A.U., Akhilesh, A.G., and O.U. has performed the behavioral and molecular studies. Statistical analysis and compilation of first draft 
manuscript was done by A.U. and A.K. Finally, V.T. has edited and revised the manuscript, and prepared it for submission. The project supervision and administration were done by V.T. All authors have approved the final draft of the manuscript.

\section{Data availability}

The datasets generated during and/or analyzed during the current study are available from the corresponding author on reasonable request.

\section{References}

1. Aboulmaouahib S, Madkour A, Kaarouch I et al (2018) Impact of alcohol and cigarette smoking consumption in male fertility potential: Looks at lipid peroxidation, enzymatic antioxidant activities and sperm DNA damage. Andrologia 50:e12926

2. Ahadi S, Zargari M, Khalatbary AR (2019) Assessment of the neuroprotective effects of (-)epigallocatechin-3-gallate on spinal cord ischemia-reperfusion injury in rats. J Spinal Cord Med 1-8

3. Ahmad A, Ali T, Rehman SU, Kim MO (2019) Phytomedicine-based potent antioxidant, Fisetin protects CNS-Insult LPS-induced oxidative stress-mediated neurodegeneration and memory impairment. J Clin Med 8:850

4. Ambade A, Mandrekar P (2012) Oxidative Stress and Inflammation: Essential Partners in Alcoholic Liver Disease. Int J Hepatol. https://doi.org/10.1155/2012/853175

5. Bhatia S, Drake DM, Miller L, Wells PG (2019) Oxidative stress and DNA damage in the mechanism of fetal alcohol spectrum disorders. Birth defects Res 111:714-748

6. Butterfield DA, Boyd-Kimball D (2020) Mitochondrial Oxidative and Nitrosative Stress and Alzheimer Disease. Antioxidants 9:818

7. Castillo-Carniglia A, Keyes KM, Hasin DS, Cerdá M (2019) Psychiatric comorbidities in alcohol use disorder. The Lancet Psychiatry 6:1068-1080

8. Claiborne A (2018) Catalase activity. In: Handbook Methods for Oxygen Radical Research

9. Crespi C, Galandra C, Canessa N et al (2020) Microstructural damage of white-matter tracts connecting large-scale networks is related to impaired executive profile in alcohol use disorder. Neurolmage Clin 25:102141

10. Crespi C, Galandra C, Manera M et al (2019) Executive impairment in Alcohol Use Disorder reflects structural changes in large-scale brain networks: A joint Independent Component Analysis on greymatter and white-matter features. Front Psychol 10:2479

11. Crichton GE, Bryan J, Murphy KJ (2013) Dietary antioxidants, cognitive function and dementia-a systematic review. Plant foods Hum Nutr 68:279-292

12. Dwivedi DK, Kumar D, Kwatra M et al (2018) Voluntary alcohol consumption exacerbated high fat diet-induced cognitive deficits by NF-KB-calpain dependent apoptotic cell death in rat hippocampus: ameliorative effect of melatonin. Biomed Pharmacother 108:1393-1403 
13. Ellman GL, Courtney KD, Andres V, Featherstone RM (1961) A new and rapid colorimetric determination of acetylcholinesterase activity. Biochem Pharmacol. https://doi.org/10.1016/00062952(61)90145-9

14. Fan AZ, Chou SP, Zhang $\mathrm{H}$ et al (2019) Prevalence and correlates of past-year recovery from DSM-5 alcohol use disorder: results from National Epidemiologic Survey on Alcohol and Related ConditionsIII. Alcohol Clin Exp Res 43:2406-2420

15. Fernandes J, Gupta GL (2019) N-acetylcysteine attenuates neuroinflammation associated depressive behavior induced by chronic unpredictable mild stress in rat. Behav Brain Res 364:356-365

16. Fukai T, Ushio-Fukai M (2020) Cross-Talk between NADPH Oxidase and Mitochondria: Role in ROS Signaling and Angiogenesis. Cells 9:1849

17. Gandhi S, Abramov AY (2012) Mechanism of oxidative stress in neurodegeneration. Oxid. Med. Cell. Longev

18. Girard M, Malauzat D, Nubukpo P (2019) Serum inflammatory molecules and markers of neuronal damage in alcohol-dependent subjects after withdrawal. World J Biol Psychiatry 20:76-90

19. He Q, Bao L, Zimering J et al (2015) The protective role of (-)-epigallocatechin-3-gallate in thrombininduced neuronal cell apoptosis and JNK-MAPK activation. Neuroreport 26:416

20. Hernández JA, López-Sánchez RC, Rendón-Ramírez A (2016) Lipids and oxidative stress associated with ethanol-induced neurological damage. Oxid Med Cell Longev 2016

21. Iliev A, Traykov V, Pradanov D et al (1999) Effect of the acetylcholinesterase inhibitor galanthamine on learning and memory in prolonged alcohol intake rat model of acetylcholine deficit. Methods Find Exp Clin Pharmacol 21:297-302

22. Islam MT (2017) Oxidative stress and mitochondrial dysfunction-linked neurodegenerative disorders. Neurol Res 39:73-82

23. Jollow D, Mitchell JR, Zampaglione N, Gillette JR (1974) Bromobenzene-induced liver necrosis. Protective role of glutathione and evidence for 3,4-bromobenzene oxide as the hepatotoxic metabolite. Pharmacology 11:151-169. https://doi.org/10.1159/000136485

24. Kono Y (1978) Generation of superoxide radical during autoxidation of hydroxylamine and an assay for superoxide dismutase. Arch Biochem Biophys. https://doi.org/10.1016/0003-9861(78)90479-4

25. Kuhad A, Bishnoi M, Tiwari V, Chopra K (2009) Suppression of NF-K $\beta$ signaling pathway by tocotrienol can prevent diabetes associated cognitive deficits. Pharmacol Biochem Behav. https://doi.org/10.1016/j.pbb.2008.12.012

26. Liu Y, Zhang Y, Zheng X et al (2018) Galantamine improves cognition, hippocampal inflammation, and synaptic plasticity impairments induced by lipopolysaccharide in mice. J Neuroinflammation $15: 112$

27. Mende MA (2019) Alcohol in the aging brain-the interplay between alcohol consumption, cognitive decline and the cardiovascular system. Front Neurosci 13 
28. Montesinos J, Pascual M, Pla A et al (2015) TLR4 elimination prevents synaptic and myelin alterations and long-term cognitive dysfunctions in adolescent mice with intermittent ethanol treatment. Brain Behav Immun 45:233-244

29. Morris RGM, Garrud P, Rawlins JNP, O'Keefe J (1982) Place navigation impaired in rats with hippocampal lesions. Nature. https://doi.org/10.1038/297681a0

30. Park D, Choi E-K, Cho T-H et al (2020) Human Neural Stem Cells Encoding ChAT Gene Restore Cognitive Function via Acetylcholine Synthesis, $A \beta$ Elimination, and Neuroregeneration in APPswe/PS1dE9 Mice. Int J Mol Sci 21:3958

31. Petrella C, Carito V, Carere $C$ et al (2020) Oxidative stress inhibition by resveratrol in alcohol dependent mice. Nutrition 110783

32. Ramdial K, Franco MC, Estevez AG (2017) Cellular mechanisms of peroxynitrite-induced neuronal death. Brain Res Bull 133:4-11

33. Rico EP, Rosemberg DB, Dias RD et al (2007) Ethanol alters acetylcholinesterase activity and gene expression in zebrafish brain. Toxicol Lett 174:25-30

34. Sanchez-Alavez M, Nguyen W, Mori S et al (2019) Time course of microglia activation and brain and blood cytokine/chemokine levels following chronic ethanol exposure and protracted withdrawal in rats. Alcohol 76:37-45

35. Sharma K, Sharma D, Sharma M et al (2018) Astaxanthin ameliorates behavioral and biochemical alterations in in-vitro and in-vivo model of neuropathic pain. Neurosci Lett. https://doi.org/10.1016/j.neulet.2018.03.030

36. Sharma M, Gupta YK (2002) Chronic treatment with trans resveratrol prevents intracerebroventricular streptozotocin induced cognitive impairment and oxidative stress in rats. Life Sci. https://doi.org/10.1016/S0024-3205(02)02083-0

37. Simpson DSA, Oliver PL (2020) ROS Generation in Microglia: Understanding Oxidative Stress and Inflammation in Neurodegenerative Disease. Antioxidants 9:743

38. Slayday RE, Gustavson DE, Elman JA et al (2020) Interaction between Alcohol Consumption and Apolipoprotein E (ApoE) Genotype with Cognition in Middle-Aged Men. J Int Neuropsychol Soc 1-13

39. Sun W, Chen L, Zheng W et al (2017) Study of acetylcholinesterase activity and apoptosis in SHSY5Y cells and mice exposed to ethanol. Toxicology 384:33-39

40. Sun X, Li G-P, Huang P et al (2020) Gnaq Protects PC12 Cells from Oxidative Damage by Activation of Nrf2 and Inhibition of NF-kB. NeuroMolecular Med 1-10

41. Tiwari V, Chopra K (2013) Resveratrol abrogates alcohol-induced cognitive deficits by attenuating oxidative-nitrosative stress and inflammatory cascade in the adult rat brain. Neurochem Int 62:861869

42. Tiwari V, Chopra K (2011) Resveratrol prevents alcohol-induced cognitive deficits and brain damage by blocking inflammatory signaling and cell death cascade in neonatal rat brain. J Neurochem 117:678-690 
43. Tobore TO (2019) On the neurobiological role of oxidative stress in alcohol-induced impulsive, aggressive and suicidal behavior. Subst Use Misuse 54:2290-2303

44. Uniyal A, Singh R, Akhtar A et al (2019) Co-treatment of piracetam with risperidone rescued extinction deficits in experimental paradigms of post-traumatic stress disorder by restoring the physiological alterations in cortex and hippocampus. Pharmacol Biochem Behav. https://doi.org/10.1016/j.pbb.2019.172763

45. Wei JC-C, Huang H-C, Chen W-J et al (2016) Epigallocatechin gallate attenuates amyloid $\beta$-induced inflammation and neurotoxicity in EOC 13.31 microglia. Eur J Pharmacol 770:16-24

46. West RK, Maynard ME, Leasure JL (2018) Binge ethanol effects on prefrontal cortex neurons, spatial working memory and task-induced neuronal activation in male and female rats. Physiol Behav 188:79-85

47. Xicota L, Rodriguez-Morato J, Dierssen M, de la Torre R (2017) Potential role of (-)-epigallocatechin-3gallate (EGCG) in the secondary prevention of Alzheimer disease. Curr Drug Targets 18:174-195

48. Xu H, Liu D, Chen J et al (2019) Effects of Chronic Voluntary Alcohol Drinking on Thiamine Concentrations, Endoplasmic Reticulum Stress, and Oxidative Stress in the Brain of Crossed High Alcohol Preferring Mice. Neurotox Res 36:777-787

49. Xu Q, Langley M, Kanthasamy AG, Reddy MB (2017) Epigallocatechin gallate has a neurorescue effect in a mouse model of parkinson disease. J Nutr 147:1926-1931

50. Xu Y, Xie M, Xue J et al (2020) EGCG ameliorates neuronal and behavioral defects by remodeling gut microbiota and TotM expression in Drosophila models of Parkinson's disease. FASEB J 34:59315950

51. Yin Q-Q, Pei J-J, Xu S et al (2013) Pioglitazone improves cognitive function via increasing insulin sensitivity and strengthening antioxidant defense system in fructose-drinking insulin resistance rats. PLoS One 8:e59313

52. Zhang Z-X, Li Y-B, Zhao R-P (2017) Epigallocatechin Gallate attenuates $\beta$-Amyloid generation and oxidative stress involvement of PPARy in N2a/APP695 cells. Neurochem Res 42:468-480

\section{Figures}



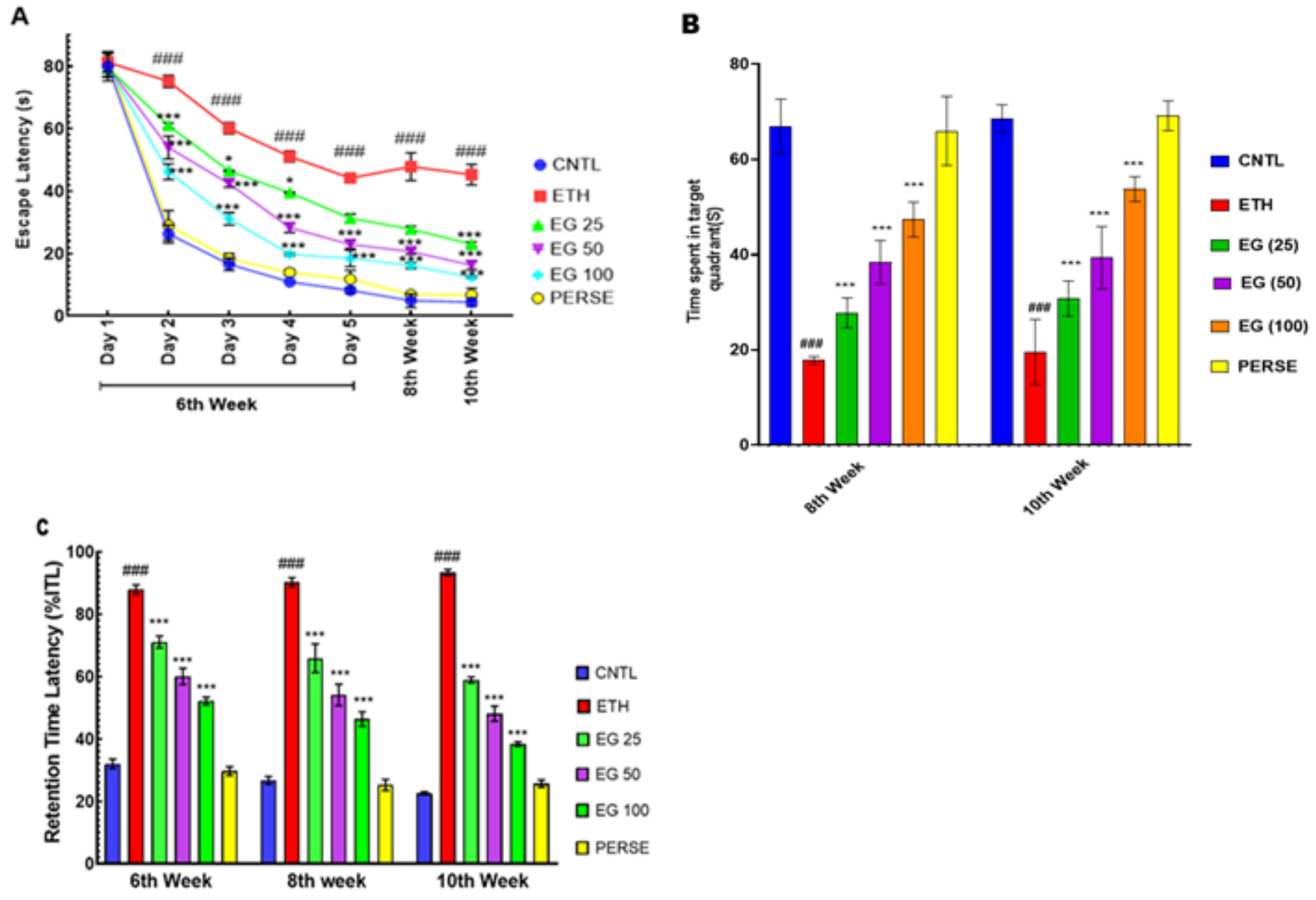

Figure 1

Chronic treatment with epigallocatechin-3-gallate $(25,50,100 \mathrm{mg} / \mathrm{kg}$; oral gavage) improves ethanol induced cognitive dysfunction. Effect on the performance of spatial memory acquisition phase in Morris water maze (A) and time spent in target quadrant in probe trial (B) in ethanol administered rats. (c) Effect of chronic treatment with epigallocatechin-3-gallate $(25,50,100 \mathrm{mg} / \mathrm{kg}$; oral gavage) on percent initial transfer latency in elevated plus maze test in ethanol administered rats. Values were expressed as mean $\pm S D$. \#\#\#P $<0.001$ represents as compared to the control group. ( $n=5$ per group). ${ }^{\star \star \star} P<0.001$ represents EGCG $(25,50$ and $100 \mathrm{mg} / \mathrm{kg})$ as compared to the ethanol treated rats. Ethanol: ETH, Epigallocatechin-3gallate dosage; EG25: 25mg/kg, EG50: 50mg/kg, EG100:100mg/kg. Two-way ANOVA followed by Tukey's multiple comparisons test analysis was used. Statistical significance was considered at $\mathrm{P}<0.05$. 

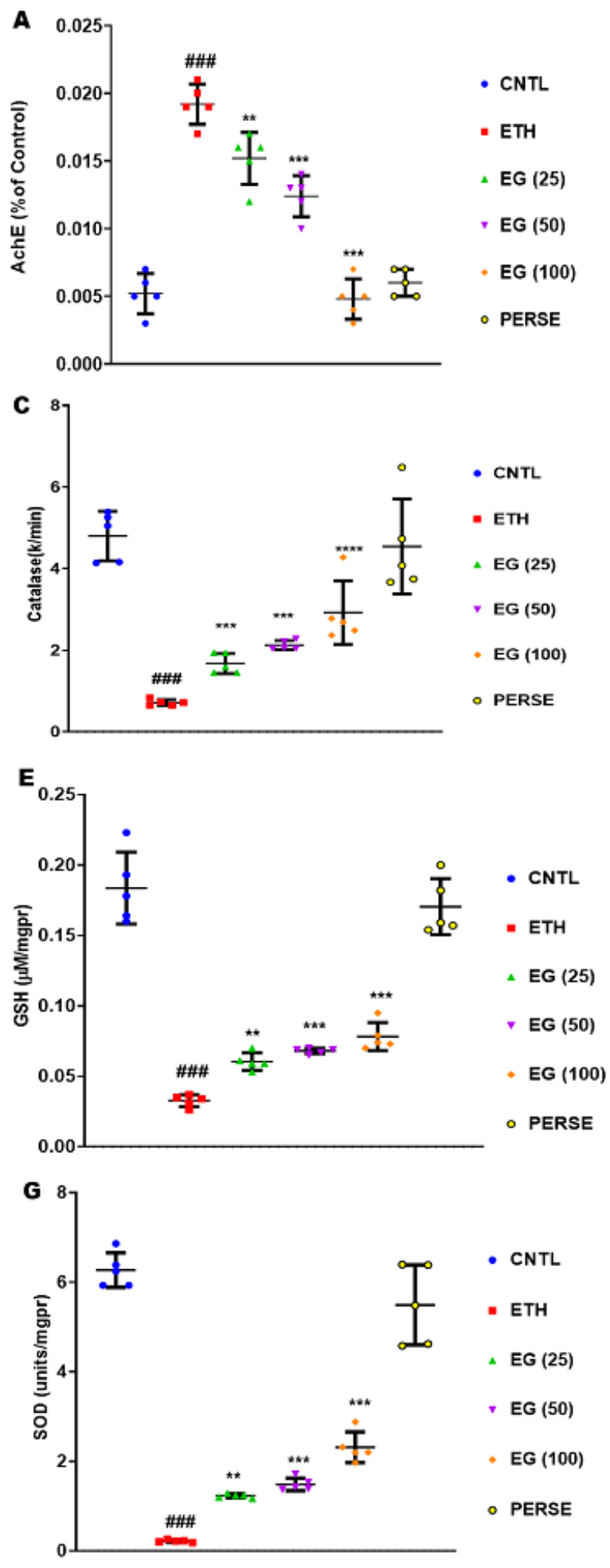
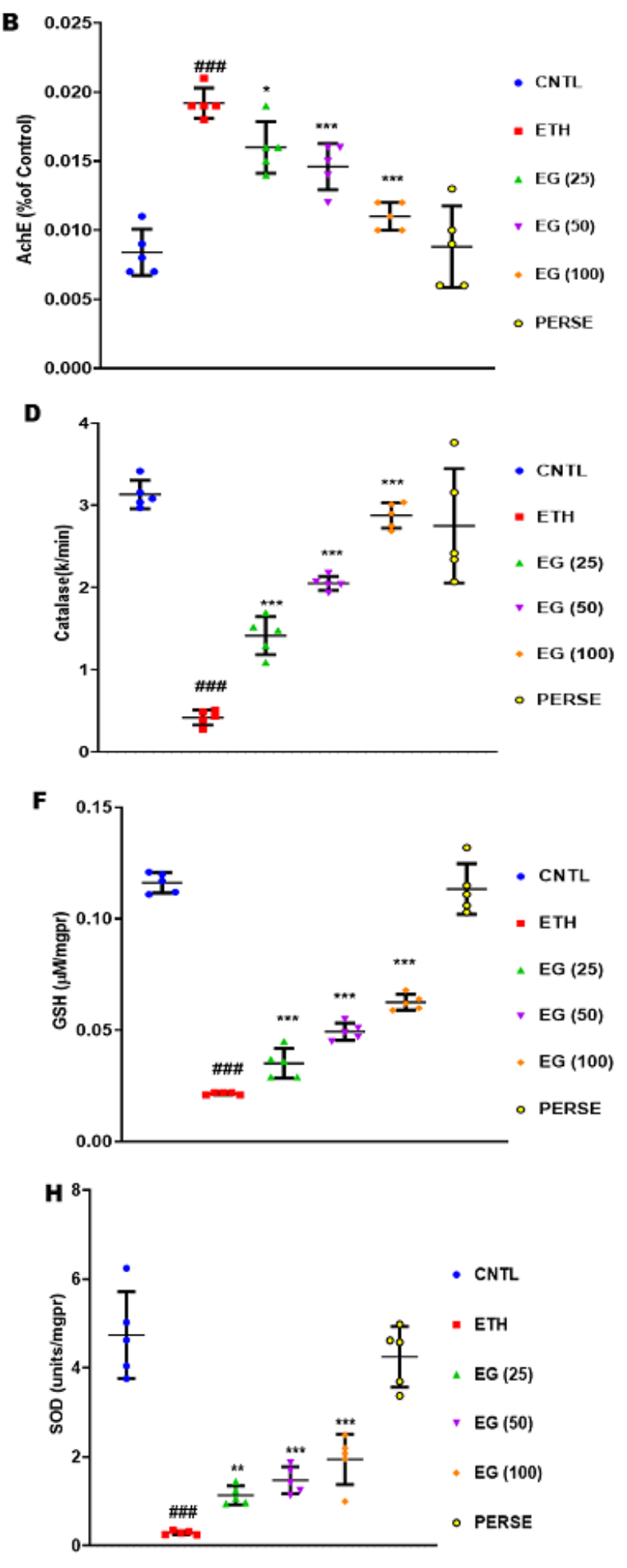

\section{Figure 2}

Epigallocatechin-3-gallate $(25,50,100 \mathrm{mg} / \mathrm{kg}$; oral gavage) treatment reduces the acetylcholinesterase activity in cerebral cortex (A) and hippocampus (B) of ethanol administered neonatal rats. EGCG treatment improves the catalase activity in cortex (C) and hippocampus (D). Chronic treatment with epigallocatechin-3-gallate $(25,50,100 \mathrm{mg} / \mathrm{kg}$; oral gavage) restores SOD levels (E: Cortex; F:

Hippocampus) and GSH (G: Cortex; H: Hippocampus). (n=5 per group). Values were expressed as mean 
$\pm S D$. \#\#\#P $<0.001$ represents as compared to the control group. *** $<0.001$ represents EGCG $(25,50$ and $100 \mathrm{mg} / \mathrm{kg}$ ) as compared to the ethanol treated rats. ( $\mathrm{n}=5-8$ per group). Ethanol: ETH, Epigallocatechin-3-gallate dosage; EG25: 25mg/kg, EG50: 50mg/kg, EG100:100mg/kg. One-way ANOVA followed by Bartlett's test analysis was used. Statistical significance was considered at $P<0.05$.
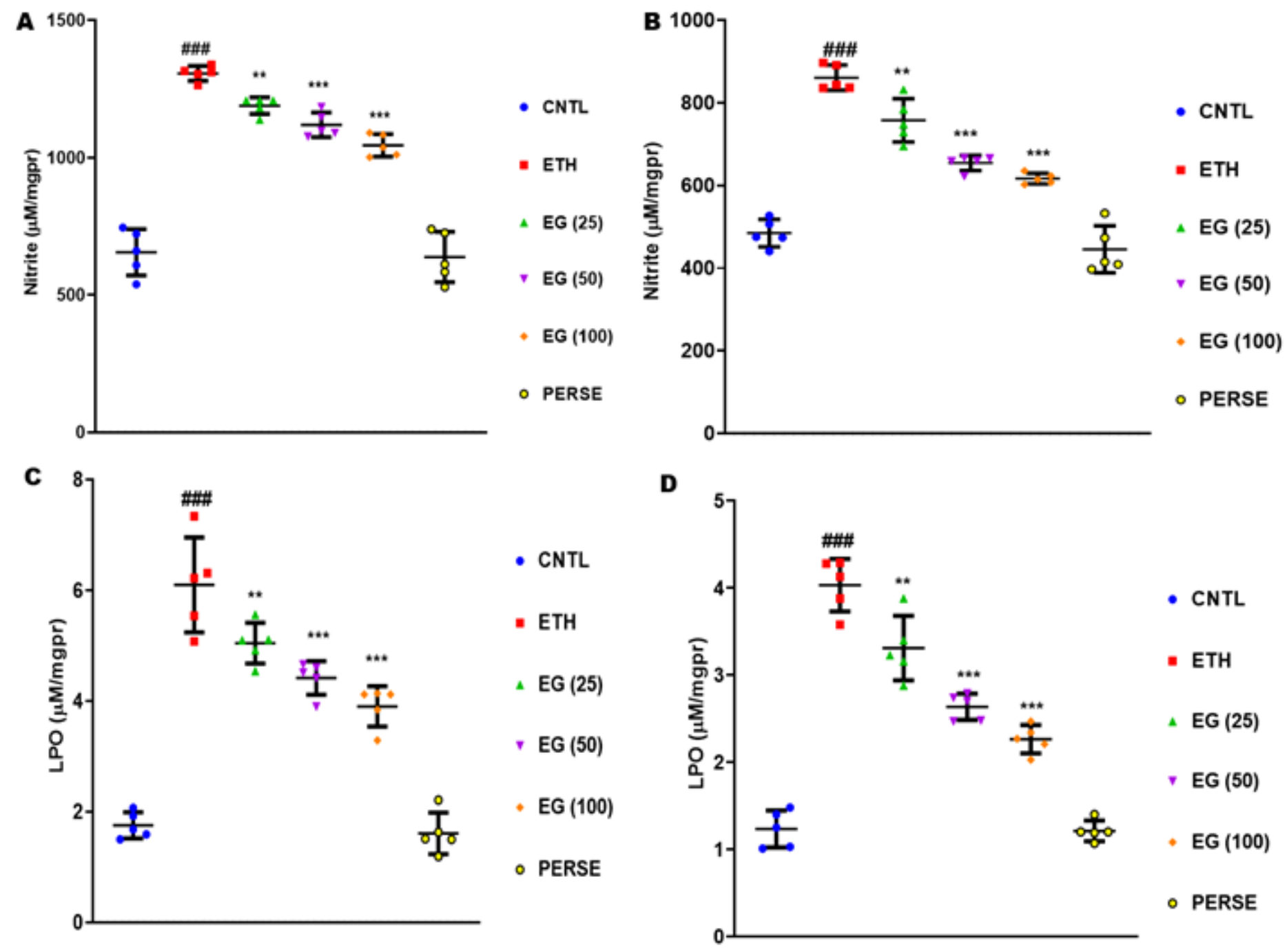

Figure 3

Epigallocatechin-3-gallate (25, 50, $100 \mathrm{mg} / \mathrm{kg}$; oral gavage) suppressed nitrite levels (A: cortical and B: hippocampal) and LPO (C: cortical and D: hippocampal) in rats chronically administered ethanol. ( $\mathrm{n}=5$ per group). Values were expressed as mean \pm S.D. \#\#\#P $<0.001$ represents as compared to the control group. ${ }^{*} * \mathrm{P}<0.001$ represents $\mathrm{EGCG}(25,50$ and $100 \mathrm{mg} / \mathrm{kg})$ as compared to the ethanol treated rats. One-way ANOVA followed by Bartlett's test analysis was used. Statistical significance was considered at $\mathrm{P}<0.05$. 

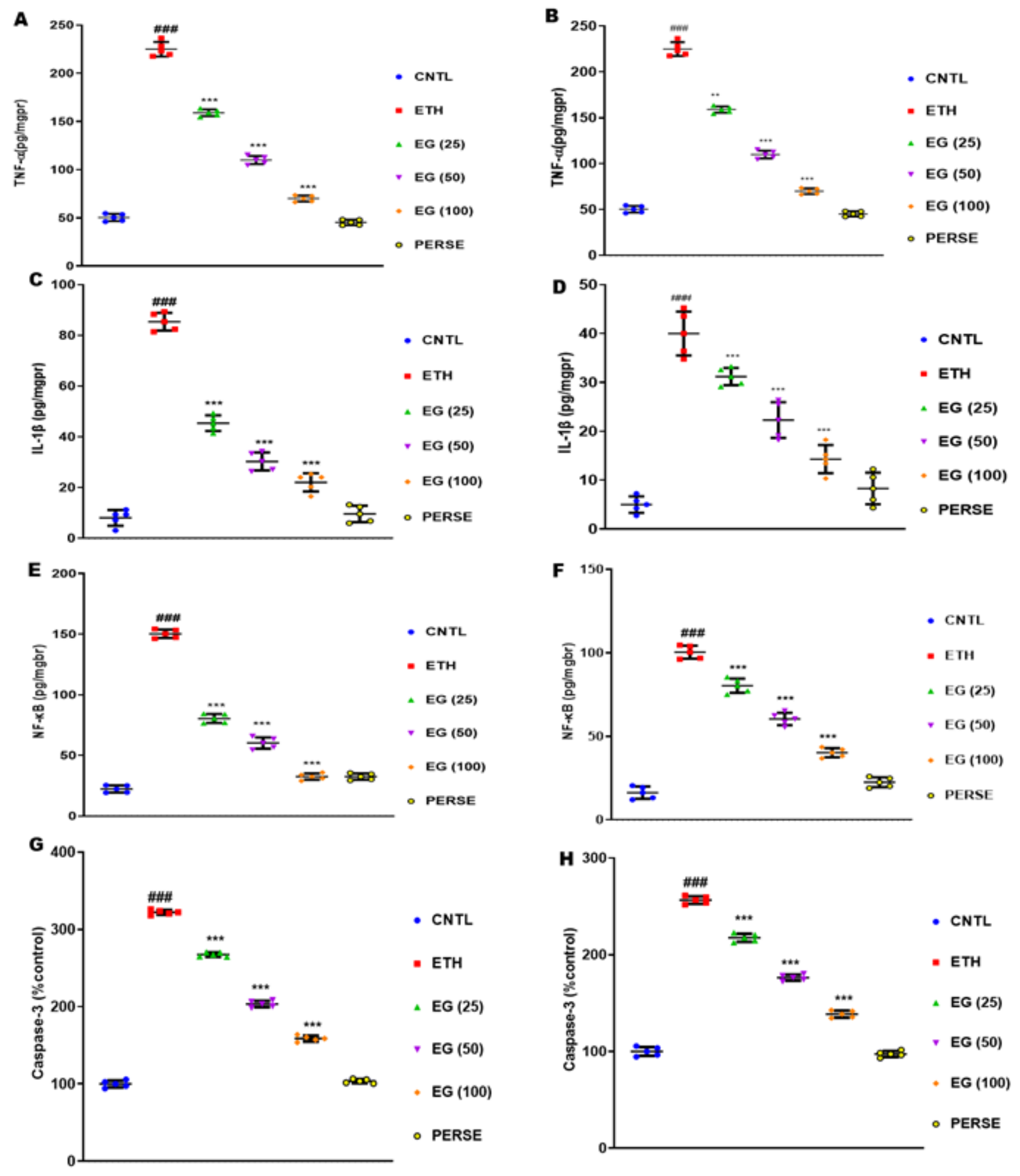

Figure 4

Epigallocatechin-3-gallate (25, 50, $100 \mathrm{mg} / \mathrm{kg}$; oral gavage) suppressed TNF-a levels (A: Cortex and B: Hippocampus) and IL-1 $\beta$ (C: Cortex and D: Hippocampus) in rats chronically administered ethanol. Chronic treatment with epigallocatechin-3-gallate $(25,50,100 \mathrm{mg} / \mathrm{kg}$; oral gavage) reduced NF-K $\beta$ (E: Cortex and F: Hippocampus) and caspase-3 (G: Cortex and H: Hippocampus) levels in ethanol administered rats. Values were expressed as mean \pm S.D. ( $n=5-8$ per group). Values were expressed as 
mean \pm SD \#\#\#P $<0.001$ represents as compared to the control group. $* \star \star P<0.001$ represents EGCG $(25$, 50 and $100 \mathrm{mg} / \mathrm{kg}$ ) as compared to the ethanol treated rats. One-way ANOVA followed by Bartlett's test analysis was used. Statistical significance was considered at $\mathrm{P}<0.05$.

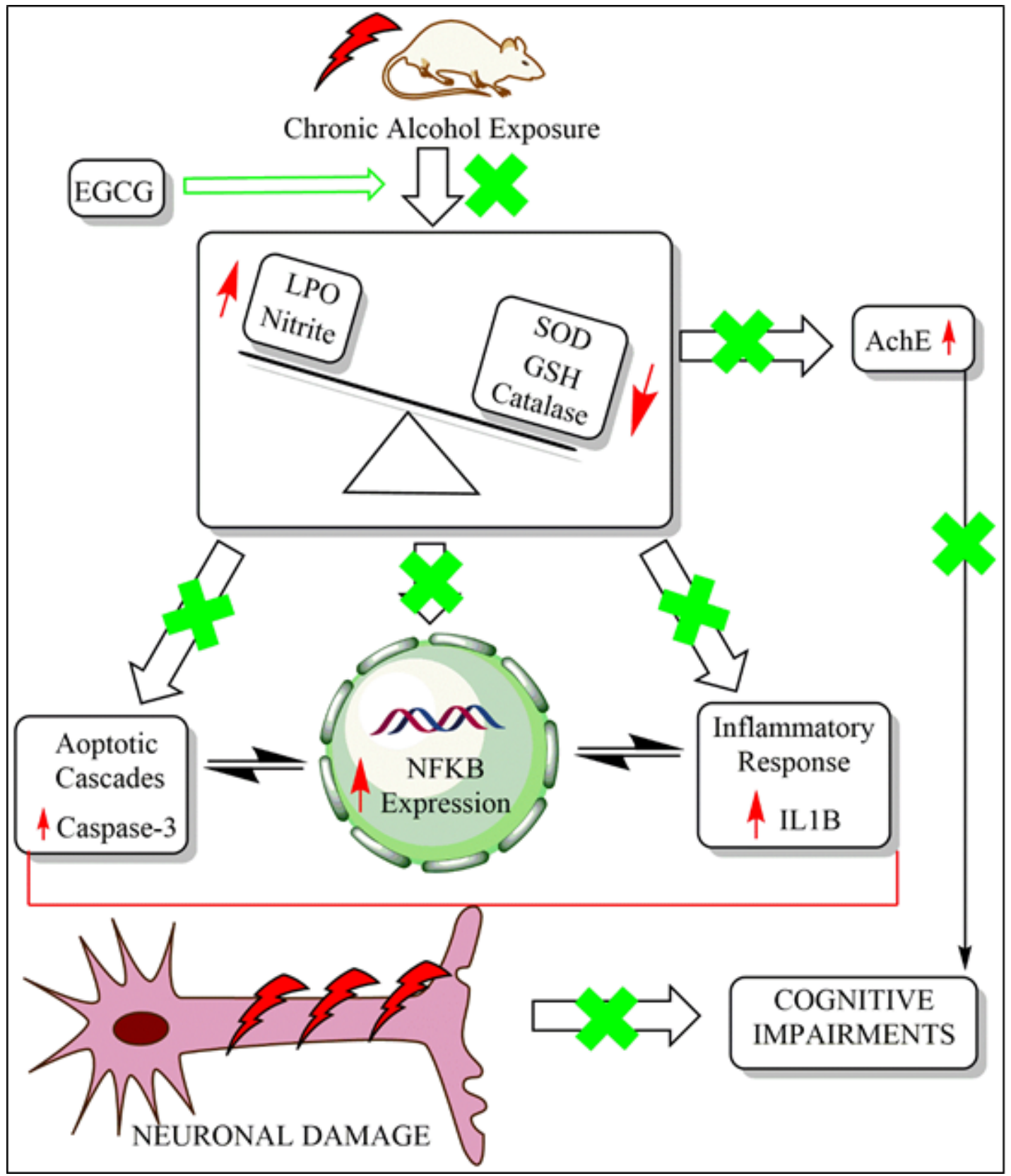

Figure 5

Summary of findings. The chronic alcohol exposure activates oxido-nitrosative stress pathways crosslinked with inflammation and cell death cascades. This, further leads to the neuronal damage that precipitates the cognitive dysfunction. The EGCG treatment reverses these pathophysiological aberrations and improves cognitive functioning. 